sequelae and their relationship to increased HIV transmission. CDC guidelines advocate testing MSM at least annually for these infections, but surveys of medical providers suggest that adherence to these guidelines is minimal. Because providers cite limited time and staff as common reasons for not following the guidelines, we evaluated the feasibility and accuracy of performing self-administered testing for GC and CT.

Methods 286 clients who attended Whitman-Walker Clinic in Washington, DC for HIV/STI testing participated in the study. Enrolled clients had a mean age of $36 \pm 11$, represented a variety of racial/ethnic backgrounds with $52.8 \%$ identifying as Caucasian, and had an average of two male partners in the last 30 days. Clients performed screening using the GenProbe APTIMA 2 Combo (AC2) kit after viewing written and pictorial instructions. A trained provider also performed the testing with the order of client vs provider randomised to adjust for any training effect. This provider remained in the room while the client performed screening to observe, but did not provide assistance.

Results The overall prevalence of GC and CT in this sample was $8.9 \%$ for P-GC, $8.5 \%$ for R-GC, $1.77 \%$ for P-CT, and $13.3 \%$ for R-CT. $\mathrm{McNemar}$ tests were performed stratified by type of infection and anatomic site to evaluate concordance of the client vs provider results. Clients were found to be significantly better at identifying PGC $(91.3 \%$ vs $94.4 \% ; 8.8 \%$ vs $5.6 \% ; \mathrm{p}=0.01)$ and R-GC $(91.5 \%$ vs $94.3 \% ; 8.5 \%$ vs $5.7 \% ; \mathrm{p}=0.03)$ and to have results equivalent to providers for P-CT $(98.3 \%$ vs $98.9 \% ; 1.8 \%$ vs $1.1 \%$; $=0.50)$ and RCT $(88.7 \%$ vs $88.2 \%$; $13.3 \%$ vs $11.9 \%, p=0.25)$ detection.

Conclusions The positive predictive value of the AC2 test makes it unlikely that clients obtained false positives, and observation of subjects while they performed screening ruled out cross-contamination of samples. Therefore, the higher detection rate among the clients is most likely attributable to a more rigorous swabbing technique that sampled an increased surface area. These results suggest that individuals are capable of performing their own STI screening and that allowing them to do so may increase infection detection rates and treatment.

\section{3-S3.05 CHLAMYDIA TRACHOMATIS DETECTION BY NUCLEIC ACID AMPLIFICATION ASSAY USING RECTAL SWABS}

\section{doi:10.1136/sextrans-2011-050109.119}

1J Williams, ${ }^{2} \mathrm{~B}$ Van Der Pol, ${ }^{1} \mathrm{~A}$ Pantone. ${ }^{1}$ Indiana University School of Medicine, Indianapolis, USA; ${ }^{2}$ Indiana University Shool of Public Health, Bloomington, USA

Background Rectal infection with C trachomatis (CT) is increasing in many settings; however, there are currently no FDA approved NAAT for use with rectal specimens. Access to reliable diagnostics using rectal specimens is critical to both surveillance and disease management and control. This is important as CT culture has been shown to have lower sensitivity, $54.8 \%$, when compared to NAAT in our patient population. The objective of this study was to evaluate the performance of rectal swabs tested on the Abbott m2000 (m2000) as compared to the GenProbe APTIMA Combo 2 (AC2) for detection of CT.

Methods Rectal samples were collected and placed into chlamydia transport medium (CTM) for testing by both m2000 and AC2 for CT. CTM was split as follows; $1 \mathrm{ml}$ into an empty m2000 tube; 100 ul into m2000 multi-collect tube containing buffer; $100 \mathrm{ul}$ into an AC2 swab collection tube. From this point forward, testing was performed according to the package insert for both platforms with two CT negative samples being tested for every positive one. $\mathrm{k}$ scores were determined to measure agreement between the $\mathrm{m} 2000$ and AC2 collection tubes.

Results A total of 59 samples were tested for CT by m2000 and AC2. AC2 was considered the reference standard for this study with
20 samples identified as positive and 39 as negative for CT. Neat CTM placed into an empty m2000 tube detected 95\% (19/20) and had a single positive that was not detected by AC2 (38/39 agreed). The single neat CTM missed by $\mathrm{m} 2000$ was positive in the spiked multi-collect tube. CTM spiked into an m2000 multi-collect tubes also detected all but one of the infections identified by AC2 (19/20) and negatives agreed completely (39/39). The m2000 multi-collect miss was CT positive in the neat sample. Both collection methods on the m2000 generated results that had very good agreement with the reference test: ? scores were 0.924 for empty and 0.962 for multicollect tubes.

Conclusion The $\mathrm{m} 2000$ has excellent performance characteristics compared to AC2 for the detection of CT. NAATs offer an alternative to culture for the detection of CT in rectal samples, and are less susceptible to transport conditions and sterility that are often a concern with culture. The collection of rectal specimens in CTM offers the opportunity for routine testing using multiple collection devices and platforms with the data suggesting that the m2000 assay can be used to meet the revised CDC recommendations for rectal testing for $\mathrm{CT}$.

\section{3-S3.06 RESCREENING FOR CHLAMYDIAL INFECTION USING HOME-BASED, SELF-OBTAINED VAGINAL SWABS: A RANDOMISED CONTROLLED TRIAL IN FAMILY PLANNING CLINIC CLIENTS}

doi:10.1136/sextrans-2011-050109.120

${ }^{1} \mathrm{~F} \mathrm{Xu},{ }^{2} \mathrm{~B}$ Stoner, ${ }^{3} \mathrm{~S}$ Taylor, ${ }^{4} \mathrm{~L}$ Mena, ${ }^{1} \mathrm{~L}$ Tian, ${ }^{1} \mathrm{~J}$ Papp, ${ }^{1} \mathrm{~K}$ Hutchins, ${ }^{3} \mathrm{D}$ Martin, ${ }^{1} \mathrm{~L}$ Markowitz. ${ }^{1}$ CDC, Atlanta, USA; ${ }^{2}$ Washington University St. Louis, USA; ${ }^{3}$ Louisiana State University Health Sciences Center, New Orleans, USA; ${ }^{4}$ University of Mississippi, Medical Center and Mississippi Jackson, USA

Background Family planning clinics provide contraceptive and preventive services for millions of low-income individuals. Screening and treatment for Chlamydia trachomatis infection in these clinics is a major part of the chlamydia control program in the USA. For women diagnosed with chlamydia, rescreening 3 months after treatment is recommended according to national guidelines. However, rescreening rates are low. The time and effort needed for patients to return to the clinic and the lack of access to follow-up care may contribute to the poor adherence to the rescreening recommendation.

Methods We conducted a randomised controlled trial in family planning clinics in three cities. After informed consent, women/girls $>16$ years treated for laboratory-confirmed chlamydial infection were randomly assigned to the Home Group (mailed a vaginal swab kit for self collection at home) or the Clinic Group (made a clinic appointment) for rescreening at 3 months following treatment. Reminder calls were made about 2 weeks before scheduled rescreening. The endpoint was rescreening within a 7 week window, 1 week before to 6 weeks after, the scheduled rescreening date.

Results 404 women were enrolled and their group assignments were randomised by opening centrally stuffed envelops. Women assigned to the Home Group had higher rescreening rate than those in the Clinic Group: Overall, 40.8\% of 196 in the Home Group and 20.7\% of 208 in the Clinic Group were rescreened $(p<0.001)$. The rescreening rates were $38.4 \%$ (Home) vs $19.8 \%$ (Clinic) among those living with parents, and $48.2 \%$ (Home) vs $21.2 \%$ (Clinic) among those with a history of chlamydia infection prior to the treated episode at enrolment (both $\mathrm{p}<0.001$ ). Among women reached by a reminder call, rescreening rates were significantly higher in the Home Group (59.2\% of 130$)$ than in the Clinic Group (37.8\% of 111$)(p<0.001)$. Among 163 women not reached by the reminder call, the rescreening rate were low $(<5 \%)$ in both groups. In the Home Group, 12 